(1)

CrossMark

\title{
Strategies for the prevention and management of coronavirus disease 2019
}

\author{
Wei-jie Guan ${ }^{1,3}$, Rong-chang Chen ${ }^{2,3}$ and Nan-shan Zhong ${ }^{1}$
}

Affiliations: 'State Key Laboratory of Respiratory Disease, National Clinical Research Center for Respiratory Disease, Guangzhou Institute of Respiratory Health, The First Affiliated Hospital of Guangzhou Medical University, Guangzhou Medical University, Guangzhou, China. ${ }^{2}$ Pulmonary and Critical Care Dept, Shenzhen Institute of Respiratory Diseases, First Affiliated Hospital of South University of Science and Technology of China (Shenzhen People's Hospital), Shenzhen, Guangdong, China. ${ }^{3}$ Wei-jie Guan and Rong-chang Chen contributed equally to the article.

Correspondence: Nan-shan Zhong, State Key Laboratory of Respiratory Disease, National Clinical Research Center for Respiratory Disease, Guangzhou Institute of Respiratory Health, The First Affiliated Hospital of Guangzhou Medical University, 151 Yanjiang Road, Guangzhou, Guangdong, China. E-mail: nanshanđavip.163.com

@ERSpublications

Early protection, early identification, early diagnosis, and early isolation are crucial to combat COVID-19 outbreaks https://bit.ly/38ZZYQg

Cite this article as: Guan W-J, Chen R-C, Zhong N-S. Strategies for the prevention and management of coronavirus disease 2019. Eur Respir J 2020; 55: 2000597 [https://doi.org/10.1183/13993003.00597-2020].

Since the outbreak in Wuhan city in December 2019, there has been a surge of newly diagnosed cases with coronavirus disease 2019 (COVID-19) globally [1-4]. The total number has reached to 101927 laboratory-confirmed cases as of 8 March, 2020 [5]. An increasing number of countries have issued alerts of the highest level.

COVID-19 is caused by infection with severe acute coronavirus 2 (SARS-CoV-2) [1]. Healthcare professionals have been confronted with several pressing challenges since the onset of outbreak. In the initial stages, most cases had direct contact with wildlife and human-to-human transmission had not yet been demonstrated. Not all hospitals had been equipped with sufficiently protective resources. Subsequently, the number of cases who had recent contact with people from Wuhan rapidly increased. Several clusters of infected individuals have been documented in cities such as Wuhan and Hong Kong [1, 6, 7], providing the initial evidence of human-to-human transmission. The rapid transmission (median $\mathrm{R}_{0} \sim 3.0$ ) could have resulted from the diverse routes of transmission, the virulence and the susceptibility of the population. An exemplar is the recent outbreak of cases (>1000 out of 3711 passengers) from the Diamond Princess cruise ship [8]. Early detection and isolation of cases have been the bedrock for curbing the rapid spread of communicable diseases. Caution should be, however, exercised to promptly identify asymptomatic viral carriers [9]. Additionally, other routes of transmission of SARS-CoV-2 (i.e. fomite transmission) might have predisposed to the rapid spread globally. SARS-CoV-2 has been detected in the gastric mucosa, stool, urine and saliva [10-12]. A press conference was immediately held to disclose the detection and isolation of the live virus from stool specimens to the general public [13]. Improved personal protection and hygiene management at community levels were then officially endorsed to protect the population from infections.

Meanwhile, the medical staff in most hospitals of Hubei province (particularly Wuhan city) suffered from burnout due to the overwhelming workload and the lack of personal protective equipment. This was aggravated by the shortage of medical resources (i.e. oxygen supply, ventilators), and therefore mechanical

Copyright $\odot$ ERS 2020. This article is open access and distributed under the terms of the Creative Commons Attribution Non-Commercial Licence 4.0. 
ventilation could not be initiated in a timely manner in all patients who had hypoxaemia. Another pressing need was to promptly discriminate patients with COVID-19 from other febrile diseases. Based on the panel of experts of respiratory medicine, microbiology and infectious diseases, and the Chinese Center for Disease Prevention and Control (CDC), the Chinese government promptly informed the public not to travel to Wuhan city, and mandated that the number of cases be reported by all local governments in real-time. The lockdown of Wuhan and several other cities in China has been shown to effectively prevent largescale transmission of cases to other regions. Security checks with body temperature assessment became mandatory for entry to communities and various public facilities. Mobile cabin hospitals have been constructed within 10 days, and more than 60000 medical staff and resources have been dispatched to Hubei province (particularly Wuhan city), empowering the local hospitals to aggregate and manage the cases. The interagency mechanism that integrates early protection, early identification, early diagnosis, and early isolation has effectively curbed the rapidly growing outbreak.

Development of new technologies are urgently needed for the diagnosis of COVID-19. Most laboratory testing has been based on viral nucleic acids assay. However, conventional laboratory testing techniques have been limited by the low diagnostic performance and the long waiting time. In light that quality control is vital to the confirmation of diagnosis, provision of reverse transcription polymerase chain reaction assay kits has been made to provincial hospitals since 23 January, 2020. Hospital staff have been trained in techniques to obtain nasopharyngeal swabs with sufficient quality. Hence, it was no longer mandated that samples for confirmatory testing would be submitted to the CDC, which helped to substantially reduce the waiting time. To better empower laboratory testing, the chip-based isothermal amplification analyser which could simultaneously detect 16 respiratory viruses (including SARS-CoV-2) within $45 \mathrm{~min}$ has been developed [14]. The rapid immunoglobin M assay kit for detecting SARS-CoV-2 with only a drop of blood $(\sim 10 \mu \mathrm{L})$ has also been developed, with the results being available within only $15 \mathrm{~min}$ and online analysis through smartphone [15]. These novel laboratory testing techniques would greatly facilitate the clinical diagnosis of COVID-19 with greater precision and results accessibility and shortened duration.

At the start of the outbreak there was little knowledge of the origins, clinical presentation or outcomes of COVID-19. Currently, more than 100 articles from China have been published within 3 months. The initial reports have revealed the possible origins and the tissue tropism of SARS-CoV-2 [16, 17], the clinical characteristics of patients admitted in Wuhan city [1, 2, 18-20], the transmission dynamics of outbreak [7] and the modelling of the outbreak [21]. These have been valuable to inform the public the potential of human-to-human transmission. Subsequent reports have documented the clinical characteristics of patients from a nationwide cohort [12], among the critically ill patients [22] and among those managed outside Wuhan city [23-26], the dynamic changes in the radiological manifestations [27], and the susceptibility of infants [28] and pregnant women [29]. Thanks to these publications, it becomes clear that nearly half of patients with COVID-19 could be afebrile, that an absence of radiological abnormality could be found among symptomatic individuals on hospital admission, that lymphopenia, high lactate dehydrogenase and direct bilirubin were prominent laboratory abnormalities, and that vertical transmission was unlikely.

Unfortunately, no targeted therapy exists for SARS-CoV-2 infections. Past experience of SARS and MERS has been used to guide clinical practice. Supportive therapies (i.e. oxygen supplementation, antibiotics) have been implemented with modest outcomes. Although several antiviral medications (i.e. remdesivir, chloroquine, loparinir/ritonavir) have been adopted for compassionate therapy without clear evidence of benefit, and a randomised controlled trial with remdesivir has been initiated (NCT04252664), no results of randomised controlled trials are available. Given the rapid spreading of new cases, it was challenging to organise well-designed clinical trials within such a short time. Hitherto, more than 150 clinical trials have been initiated in China and the government has established regulations to coordinate these trials. While chloroquine appeared to be superior to arbidol and loparinir/ritonavir in terms of shortening the duration to negative testing of viral RNA, a number of traditional Chinese medicines might be promising. Lianhuaqingwen capsules and Liushen capsules have been shown to inhibit viral replication in vitro (Z.F. Yang and co-workers, unpublished data). A pilot study with convalescent plasma from patients with COVID-19 has revealed promising therapeutic outcomes in terms of rapidly rendering viral RNA testing negative and improving oxygen saturation (K. Duan and co-workers, unpublished data). We have recently identified significant patient-ventilator asynchrony among the critically ill patients, with the underlying causes being unclear. Airway morphologic evaluation with optical coherence tomography (which could probe distal airways up to the ninth generation of bronchi) did not reveal significant airway remodelling (including severe obstructions) (N.S. Zhong and co-workers, unpublished data). Subsequent testing revealed markedly increased levels of mucin 1 and mucin 5AC in the sputum aspirated from the trachea. This was concordant with the pathological findings from autopsy samples, indicating that apart from 
TABLE 1 Key unanswered questions of the coronavirus disease 2019 (COVID-19) outbreak

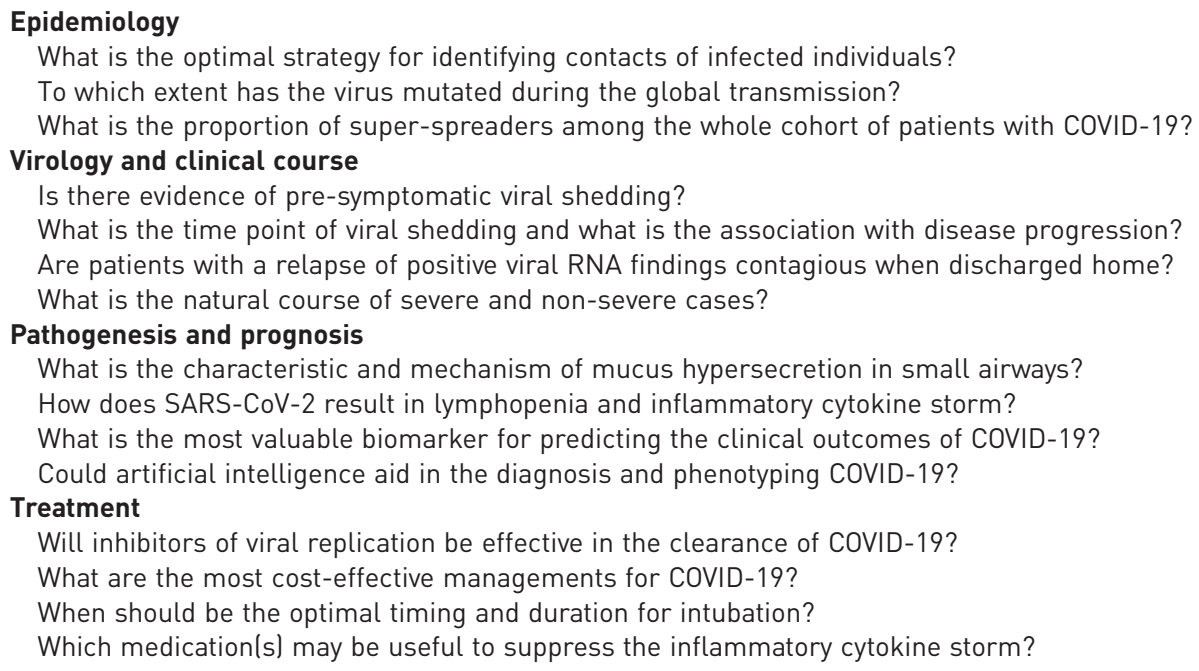

leukocyte and macrophage infiltration, microvascular infarction and interstitial fibrosis, the most defining characteristic of COVID-19 is mucus staggering in the bronchioles and alveoli [30]. Therapies that target at airway humidification and suppression of mucin secretion might help improve clinical outcomes. In light of the significantly decreased airway resistance and safety, inhalation of hydrogen and oxygen mixed gas which is generated through water electrolysis has been applied in clinical practice. Hydrogen/oxygen mixed gas inhalation resulted in a major amelioration of dyspnoea in most patients with COVID-19 in a pilot investigation, and has therefore been endorsed by the latest Recommendation for the Diagnosis and Management of Covid-19 document [31].

From the public health standpoint, disease control would benefit from the derivation of an accurate prediction model that, unlike previously published models, takes into account the impact of governmental interventions, the diversity of human-to-human transmission, and the massive domestic migration around the Spring Festival. Few models exist that could be adapted to the conditions within mainland China well. The latest modified susceptible-exposed-infectious-removed and artificial intelligence predictive model has successfully heralded the trend of changes according to the Chinese official daily announcement [32]. Importantly, the model accurately predicted the changes inside and outside Wuhan city and Hubei province. Therefore, newly developed prediction models of the COVID-19 outbreak need to be adapted to the local conditions.

Despite the rapidly accumulating knowledge, there remain many unanswered questions that await further investigations (table 1). These included the precise routes of transmission, the host tropisms, viral load dynamics, host defence responses, and the optimal therapeutic management for COVID-19. Recently, we have identified some patients with a relapse of positive viral RNA findings when discharged home. Although these patients were unlikely to have experienced a relapse of COVID-19 (after the formation of specific antibodies to SARS-CoV-2), it remains to be determined whether they are sources of transmission or should be quarantined. As the outbreak is rapidly spreading globally, we hope that our experience would help inform medical staff and contribute to the effective control of the outbreak worldwide.

Acknowledgement: We thank W. Ian Lipkin (Mailman School of Public Health, Columbia University, New York, NY, USA) for his insightful suggestions.

Author contributions: Wei-jie Guan, Rong-chang Chen and Nan-shan Zhong drafted the manuscript, provided critical review of the manuscript and approved the final draft for publication.

Conflict of interest: Wei-jie Guan has nothing to disclose. Rong-chang Chen has nothing to disclose. Nan-shan Zhong reports grants from National Health Commission, National Natural Science Foundation and Department of Science and Technology of Guangdong Province, during the conduct of the study.

Support statement: Supported by National Health Commission, National Natural Science Foundation, Department of Science and Technology of Guangdong Province. Funding information for this article has been deposited with the Crossref Funder Registry. 


\section{References}

1 Zhu N, Zhang D, Wang W, et al. A novel coronavirus from patients with pneumonia in China, 2019. $N$ Engl J Med 2020; 382: 727-733.

2 Huang C, Wang Y, Li X, et al. Clinical features of patients with 2019 novel coronavirus in Wuhan, China. Lancet 2020; 395: 497-506.

3 Holshue ML, DeBolt C, Lindquist S, et al. First case of 2019 novel coronavirus in the United States. $N$ Engl J Med 2020; 382: 929-936.

4 Wang C, Horby PW, Hayden FG, et al. A novel coronavirus outbreak of global health concern. Lancet 2020; 395: 470-473.

5 World Health Organization. Novel Coronavirus (2019-nCoV) Situation Reports. www.who.int/emergencies/ diseases/novel-coronavirus-2019/situation-reports/. Date last assessed: 11 March, 2020.

6 Chan JF, Yuan S, Kok KH, et al. A familial cluster of pneumonia associated with the 2019 novel coronavirus indicating person-to-person transmission: a study of a family cluster. Lancet 2020; 395: 514-523.

7 Li Q, Guan X, Wu P, et al. Early transmission dynamics in Wuhan, China, of novel coronavirus-infected pneumonia. N Engl J Med 2020; in press [https://doi.org/10.1056/NEJMoa2001316].

8 Xinhua News. 2 Passengers from Virus-hit Diamond Princess Cruise Ship Die in Japan. www.xinhuanet.com/ english/asiapacific/2020-02/20/c_138802347.htm. Date last accessed: 11 March, 2020.

9 Rothe C, Schunk M, Sothmann P, et al. Transmission of 2019-nCoV infection from an asymptomatic contact in Germany. N Engl J Med 2020; 382: 970-971.

10 Xie C, Jiang L, Huang G, et al. Comparison of different samples for 2019 novel coronavirus detection by nucleic acid amplification tests. Int J Infect Dis 2020; in press [https://doi.org/10.1016/j.ijid.2020.02.050].

11 To KK, Tsang OT, Chik-Yan Yip C, et al. Consistent detection of 2019 novel coronavirus in saliva. Clin Infect Dis 2020; in press [https://doi.org/10.1093/cid/ciaa149].

12 Guan WJ, Ni ZY, Hu Y, et al. Clinical characteristics of coronavirus disease 2019 in China. N Engl J Med 2020; in press [https://doi.org/10.1056/NEJMoa2002032].

13 Xinhua Net. Successful Isolation of SARS-CoV-2 from Stool Specimens by the Research Team of Prof. Nan-shan Zhong and Lan-juan Li. http://m.xinhuanet.com/gd/2020-02/13/c_1125570540.htm [Article in Chinese] Date last accessed: 11 March, 2020.

14 CapitalBio Technology. Solution for the Detection of Pathogens of Infectious Diseases. www.capitalbiotech.com/ Territory.html?id=158 [Article in Chinese] Date last accessed: 11 March, 2020.

15 State Key Laboratory of Respiratory Disease. The State Key Laboratory has Jointly Developed an Assay Kit for the Rapid Detection of Novel Coronavirus IgM. www.sklrd.cn/show.php?id=1376 [Article in Chinese] Date last accessed: 11 March, 2020.

16 Wu F, Zhao S, Yu B, et al. A new coronavirus associated with human respiratory disease in China. Nature 2020 579: 265-269.

17 Zhou P, Yang XL, Wang XG, et al. A pneumonia outbreak associated with a new coronavirus of probable bat origin. Nature 2020; 579: 270-273.

18 Chen N, Zhou M, Dong X, et al. Epidemiological and clinical characteristics of 99 cases of 2019 novel coronavirus pneumonia in Wuhan, China: a descriptive study. Lancet 2020; 395: 507-513.

19 Chang Lin M, Wei L, et al. Epidemiologic and clinical characteristics of novel coronavirus infections involving 13 patients outside Wuhan, China. JAMA 2020; in press [https://doi.org/10.1001/jama.2020.1623].

20 Wang $\mathrm{D}, \mathrm{Hu} \mathrm{B}, \mathrm{Hu} \mathrm{C}$, et al. Clinical characteristics of 138 hospitalized patients with 2019 novel coronavirusinfected pneumonia in Wuhan, China. JAMA 2020; in press [https://doi.org/10.1001/jama.2020.1585]

21 Wu JT, Leung K, Leung GM. Nowcasting and forecasting the potential domestic and international spread of the 2019-nCoV outbreak originating in Wuhan, China: a modelling study. Lancet 2020; 395: 689-697.

22 Yang X, Yu Y, Xu J, et al. Clinical course and outcomes of critically ill patients with SARS-CoV-2 pneumonia in Wuhan, China: a single-centered, retrospective, observational study. Lancet Respir Med 2020; in press [https://doi. org/10.1016/S2213-2600(20)30079-5].

$23 \mathrm{Xu}$ XW, Wu XX, Jiang XG, et al. Clinical findings in a group of patients infected with the 2019 novel coronavirus (SARS-Cov-2) outside of Wuhan, China: retrospective case series. BMJ 2020; 368: m606.

24 Zhang S, Li H, Huang S, et al. High-resolution computed tomography features of 17 cases of coronavirus disease 2019 in Sichuan province, China. Eur Respir J 2020; in press [https://doi.org/10.1183/13993003.00334-2020].

25 Wang L, Gao YH, Iou L, et al. The clinical dynamics of 18 cases of COVID-19 outside of Wuhan, China. Eur Respir J 2020; in press [https://doi.org/10.1183/13993003.00398-2020].

26 Yao Y, Tian Y, Zhou J, et al. Epidemiological characteristics of SARS-CoV-2 infections in Shaanxi, China by 8 February 2020. Eur Respir J 2020; in press [https://doi.org/10.1183/13993003.00310-2020].

27 Shi H, Han X, Jiang N, et al. Radiological findings from 81 patients with COVID-19 pneumonia in Wuhan, China: a descriptive study. Lancet Infect Dis 2020; in press [https://doi.org/10.1016/S1473-3099(20)30086-4].

28 Wei M, Yuan J, Liu Y, et al. Novel coronavirus infection in hospitalized infants under 1 year of age in China. JAMA 2020; in press [https://doi.org/10.1001/jama.2020.2131].

29 Chen H, Guo J, Wang C, et al. Clinical characteristics and intrauterine vertical transmission potential of COVID-19 infection in nine pregnant women: a retrospective review of medical records. Lancet 2020; 395 ; $809-815$

$30 \mathrm{Xu} \mathrm{Z}$, Shi L, Wang Y, et al. Pathological findings of COVID-19 associated with acute respiratory distress syndrome. Lancet Respir Med 2020; in press [https://doi.org/10.1016/S2213-2600(20)30076-X].

31 National Health Commission. Digest of the 7th Version of Guidelines for the Diagnosis and Management of Covid-19. www.nhc.gov.cn/yzygj/s7652m/202003/a31191442e29474b98bfed5579d5af95.shtml [Article in Chinese] Date last accessed: 11 March, 2020.

32 Yang Z, Zeng Z, Wang K, et al. Modified SEIR and AI prediction of the epidemics trend of Covid-19 in China under public health interventions. J Thorac Dis 2020; 12: 165-174. 